\title{
Challenges and Responsibilities of Community Workers in The New Global Coronavirus Outbreak
}

\author{
Qingqing Shan ${ }^{1}$ \\ ${ }^{1}$ Chengdu Integrated TCM and Western Medicine Hospital
}

April 28, 2020

\begin{abstract}
The outbreak of the 2019-nCoV virus has coincided with Chinese Lunar New Year, when tens of millions of people travel for the Spring Festival holiday during the world's largest annual human migration. Travellers give wings to novel coronavirus. Thanks to China's efforts that they have effectively contained the cross-border spread of the 2019-nCoV.Medicine staff make great effort to prevent and control the epidemic disease, and so do community service workers. AS of Sunday, 53 community workers had died in the line of duty in China's prevention and control of the 2019-nCoV epidemic, according to the Ministry of Civil Affairs. In the fight against 2019-nCoV, community workers have been facing enormous pressure, including a high risk of infection and inadequate protection from contamination, overwork, frustration, patients with negative emotions, a lack of contact with their families, and exhaustion. Thanks to community workers, the virus transmission route is cut off and the emergence of new cases is effectively curbed.
\end{abstract}

\section{Challenges and Responsibilities ofCommunity Workers}

in The New Global Coronavirus Outbreak

Qingqing Shan

Running title: Community Workers in The Epidemic

Authorship: Chengdu First hosp, Dept Resp Med, Chengdu 610041, Sichuan, Peoples R China

Correspondence to Qingqing Shan, Chengdu First People's Hospital, No.18, Wangxiang North Road, HighTech Zone, Chengdu, Sichuan Province, Peoples R China; Shanqingqing52377@126.com

Contributors : Shan QQ developed the concept for the manuscript and was responsible for all writing.

Ethics Statement: No ethical approval.

Conflict of Interest Statement : None declared

Data Availability Statement: No data, models, or code were generated or used during the study.

Summary: In this article, we evaluated the challenges and responsibilities of community workers in the new global coronavirus outbreak. As we are known that medical staff are saving lives, while community workers are providing a variety of services to the residents which is significantly critical to ensure early detection, early reporting, early diagnosis, early isolation, and early treatment of the disease. In China where the 2019 nCOV outbreak is being brought under control, community work has played a huge role. The sharing of these experiences is expected to help other countries in the outbreak.

Keywords: Challenges and Responsibilities, Community Workers, New Coronavirus Outbreak 
Main Text: On 29 December 2019 clinicians in a hospital in Wuhan City, China noticed a clustering of cases of unusual pneumonia. Within 4 weeks, by 26 January 2020, the causative organism had been identified as a novel coronavirus $(2019-\mathrm{nCoV})$ (Bassetti, Vena, \& Giacobbe, 2020)(Bassetti, Vena, \& Giacobbe, 2020 ${ }^{[1]}$. Overall, 196,979 cases of 2019-nCoV infection have been diagnosed in more than 100 worldwide countries ${ }^{[2]}$.

The outbreak of the 2019-nCoV virus has coincided with Chinese Lunar New Year, when tens of millions of people travel for the Spring Festival holiday during the world's largest annual human migration. Travellers give wings to novel coronavirus. Thanks to China's efforts that they have effectively contained the crossborder spread of the 2019-nCoV. (Wilson \& Chen, 2020)(Wilson \& Chen, 2020) ${ }^{[3]}$

Medicine staff make great effort to prevent and control the epidemic disease, and so do community service workers. AS of Sunday, 53 community workers had died in the line of duty in China's prevention and control of the 2019-nCoV epidemic, according to the Ministry of Civil Affairs ${ }^{[4]}$. In the fight against 2019-nCoV, community workers have been facing enormous pressure, including a high risk of infection and inadequate protection from contamination, overwork, frustration, patients with negative emotions, a lack of contact with their families, and exhaustion. Thanks to community workers, the virus transmission route is cut off and the emergence of new cases is effectively curbed.

Community-based measures are the foundation of prevention and control. The outbreak came so fierce and last so long that tens of thousands of people in mainland China were confirmed to have the disease, and hundreds had died. 2019-nCoV is a highly contagious virus, and personnel mobility gives wings to virus. However in the case of novel coronavirus (2019-nCoV), antiviral treatment and vaccination are not available(Khan, Siddique, Ali, Xue, \& Nabi, 2020)(Khan, Siddique, Ali, Xue, \& Nabi, 2020) ${ }^{[5]}$. Thus, to contain the further spread of infection, quarantine, and limitation the mobility of confirmed and suspected cases are key factors. (Khan, Siddique, et al., 2020)(Khan, Siddique, et al., 2020) ${ }^{[5]}$ Community workers play an important role in the prevention and control of 2019-nCOV.

The community is the front line of the epidemic prevention and control. community workers on the front line follow instructions and fulfill their responsibilities to curb the contagion's spread. The mission of community workers is urgent and arduous. To prevent the further spread of infection, the Chinese government has imposed a partial or full lockdown in many cities(Khan, Ali, Siddique, \& Nabi, 2020)(Khan, Ali, Siddique, \& Nabi, 2020) ${ }^{[6]}$. Community workers have to strictly control the entrance and exit of personnel and vehicle, record all the detailed information, measure human body temperature. Community workers have to be on duty all day and all night and prohibit outsiders to enter residential community. Community workers have to carry out a thorough investigation or dragnet inspection to ensure no one to be forgotten. when suspected cases are found, community workers have to take the most comprehensive, rigorous, compulsive application of traditional public health measures (surveillance, detection, isolation of infected persons and quarantine of exposed) (Khan, Siddique, et al., 2020) (Khan, Siddique, et al., 2020) ${ }^{[5]}$. Community workers have to focus on disinfection and sterilization of garbag and improvement the sanitation of public areas. It is community workers that reduce the risk of transmission and fulfill their responsibilities to achieve full victory in the battle against the virus through solid and meticulous work.

Community workers are organized to give residents easy access to adequate daily necessities during the lockdown of communities. Since the outbreak began, many cities in China have nearly shut down completely, leaving only the bare minimum of businesses open. In order to reduce the mobility, the policy of "211" (each family may assign one family member to purchase daily supplies every two days) or "411" (each family may assign one family member to purchase daily supplies every four days) is implemented ${ }^{[7]}$. Therefore, cmmunity workers have to ensure that water, electricity and gas will not be cut off during the epidemic, as well as residents' access to daily necessities, such as vegetables, grain and meat. Cmmunity workers carry out psychological intervention and psychological counseling to reduce individuals' psychological concerns.

Community workers deliver urgent intervention to avert a psychological crisis during the novel coronavirus epidemic. Sharing false messages and little understanding of the disease may likely cause panic. Community workers step up publicity work to communicate with the public through various forms such as 
community bulletin boards, hanging slogans, WeChat public accounts, community setting broadcasts, and so on (McCloskey \& Heymann, 2020)(McCloskey \& Heymann, 2020) ${ }^{[8]}$. Community workers propagandize actively to improve prevention and treatment service network and comprehensive prevention and treatment mechanism with national coverage. Public health measures which is took by community workers, such as isolation, quarantine, social distancing and community containment, are playing pivotal roles in the novel coronavirus (2019-nCoV) outbreak. Community workers are responsible for formulating psychological intervention and providing technical guidance and supervision to create a scientific and 'no rumor' atmosphere. It is the bravery and sacrifices of community workers and their contributions to the containment of the epidemic that boosted public's confidence in winning the battle against the contagion.

Patients and suspected cases are being treated in hospitals, while most people are staying at homes to reduce population flow. Medical staff are saving lives, while community workers are providing a variety of services to the residents which is significantly critical to ensure early detection, early reporting, early diagnosis, early isolation, and early treatment of the disease. In China where the outbreak is being brought under control, community work has played a huge role. The sharing of these experiences is expected to help other countries in the outbreak.

\section{References}

[1] Bassetti, M., Vena, A., \& Giacobbe, D. R. (2020). The novel Chinese coronavirus (2019-nCoV) infections: Challenges for fighting the storm. Eur J Clin Invest, 50(3), e13209. doi:10.1111/eci.13209

[2] WHO spokesperson says coronavirus has hit over 100 countries, regions.

http://www.xinhuanet.com/english/2020-03/08/c_138855749.htm.

[3] Wilson, M. E., \& Chen, L. H. (2020). Travelers Give Wings to Novel Coronavirus (2019-nCoV). J Travel Med. doi:10.1093/jtm/taaa015

[4] 53 community workers die on China's front line against epidemic.

https://www.shine.cn/news/nation/2003093777/

[5] Khan, S., Ali, A., Siddique, R., \& Nabi, G. (2020). Novel coronavirus is putting the whole world on alert. J Hosp Infect, 104(3), 252-253. doi:10.1016/j.jhin.2020.01.019

[6] Khan, S., Siddique, R., Ali, A., Xue, M., \& Nabi, G. (2020). Novel coronavirus, poor quarantine, and the risk of pandemic. J Hosp Infect. doi:10.1016/j.jhin.2020.02.002

[7] Announcement on further strengthening ten measures for epdemic prevention and control. http://www.jxstc.gov.cn/html/1072/2020-02-07/content-12079.shtml

[8] McCloskey, B., \& Heymann, D. L. (2020). SARS to novel coronavirus - old lessons and new lessons. Epidemiol Infect, 148, e22. doi:10.1017/s0950268820000254 